$16^{\text {th }}$ International Congress of Metrology, 11008 (2013)

DOI: $10.1051 /$ metrology/201311008

(c) Owned by the authors, published by EDP Sciences, 2013

\title{
Banc automatique d'étalonnage des transformateurs de courant
}

\author{
Denis Bélières ${ }^{1}$, Mohamed Agazarl ${ }^{1}$, Julien Pavard ${ }^{2}$, Daniela Istrate, Isabelle Blanc \\ ${ }^{1}$ LNE - 29 avenue Roger Hennequin - 78197 Trappes - France \\ ${ }^{2}$ IUT de Cergy Pontoise - 95 Rue Valère Collas, 95100 Argenteuil - France
}

\begin{abstract}
Current transformers allow the measurement of high AC currents (several kA) by reducing the value of this current to normalised levels of $1 \mathrm{~A}$ or $5 \mathrm{~A}$. They can therefore standardize current measurement equipements while providing dielectric isolation of the primary circuit. The parameter to be calibrated is a complex number representing the error on the ratio and the phase shift between the primary and secondary currents, which depends on the load of the transformer. This calibration can be done using a current comparator, and this method provides the best overall uncertainty (some $10^{-6}$ in relative value). Two current comparators with magnetic flux cancelling have been made in the 1970s by the Laboratoire Central des Industries Electriques. The first covers a range of primary current from $5 \mathrm{~A}$ to $1000 \mathrm{~A}$, and the second from 25 A to 25000 A. They are associated with manually operated electronic equipments: an adjustable dummy load or "burden" and an injection system for the error current. In those years, the development of the electronic devices has been very difficult due to the poor performance of the components used, and the desire to achieve a closed loop controlled system for direct reading of the current error, and direct controlling of the load. These electronic devices have been entirely redefined by abandoning the idea of a servo system for direct reading, thus complicating their operation. But they are now fully controllable by a remote computer via an USB connection, allowing full automation of the procedure: there is no more manual control, either switch or potentiometer, they all have been replaced by electronic circuits. This radical modernisation also resulted in a significant reduction of the dimensions: everything fits in a single 19" $\mathrm{x} 3 \mathrm{U}$ standard cabinet.
\end{abstract}

\section{Introduction}

Les transformateurs de courant permettent la mesure des courants élevés (plusieurs kilo-ampères) en réduisant la valeur du courant aux niveaux normalisés de $1 \mathrm{~A}$ ou $5 \mathrm{~A}$. Ils permettent donc de standardiser les équipements de mesure du courant tout en apportant une isolation diélectrique du primaire. Les paramètres à étalonner sont l'erreur sur leur rapport de transformation et le déphasage introduit entre les courants primaire et secondaire.

L'étalonnage de ces transformateurs de courant par l'emploi de comparateurs de courant est une méthode qui permet l'obtention de la meilleur incertitude globale soit quelques $10^{-6}$ en valeur relative. Deux comparateurs de courant à annulation de flux magnétique ont donc été fabriqués dans les années 1970 par le Laboratoire Central des Industries Electriques. Le premier couvre une gamme de courants primaires allant de $5 \mathrm{~A}$ à $1000 \mathrm{~A}$, et le deuxième de $25 \mathrm{~A}$ à $25000 \mathrm{~A}$. Ils sont associés à des équipements électroniques à commande manuelle: charge fictive et système d'injection de courant d'erreur.

A l'époque, la mise en œuvre de ces équipements électroniques a été très délicate en raison de la faible

\footnotetext{
${ }^{\mathrm{a}}$ Denis Bélières: denis.belieres@lne.fr
}

performance des composants utilisés, et de la volonté de réaliser un système asservi à lecture directe. En effet, cela suppose un bouclage du système électronique en contreréaction au travers de l'ensemble du comparateur de courant et du transformateur à étalonner, alors qu'ils peuvent en être éloignés de plusieurs mètres voire dizaines de mètres dans le cas d'un étalonnage sur un site industriel. Or l'exactitude de mesure demandée nécessite l'usage d'amplificateurs opérationnels à large bande passante (idéalement de plusieurs mégahertz pour des mesures à 400 hertz), et la stabilité de tels amplificateurs est incertaine lorsqu'ils sont chargés par des réactances importantes, en particulier par la capacité des câbles blindés.

Ces équipements électroniques ont été entièrement redéfinis en abandonnant l'idée d'un système asservi à lecture directe, ce qui complique leur utilisation. Mais ils sont entièrement contrôlables à distance par un ordinateur via une connexion USB, ce qui permet une automatisation complète : il n'y a plus aucune commande manuelle, que ce soit des commutateurs ou des potentiomètres, elles ont toutes été remplacées par des circuits électroniques. Cette modernisation radicale a 
également pour conséquence une réduction importante du volume (le tout tient dans un coffret standard 19 pouces $\mathrm{x}$ $3 \mathrm{U})$.

Les étendues des mesures et des charges réalisables sont clairement définies, avec sur chacune d'elles une résolution de 16 bits soit environ $2.10^{-5}$.

Un logiciel de pilotage a été développé ; il apporte une aide importante dans le choix d'une configuration parmi les nombreuses qu'offrent les deux comparateurs de courant et montre clairement les connexions à réaliser: point n'est besoin d'un quelconque manuel, et ainsi la formation du personnel appelé à utiliser ce banc devrait pouvoir se faire rapidement

\section{Les comparateurs de courant}

\subsection{Constitution du comparateur de courant}

La composition d'un comparateur de courant à flux magnétique nul est présentée dans la figure 1 .

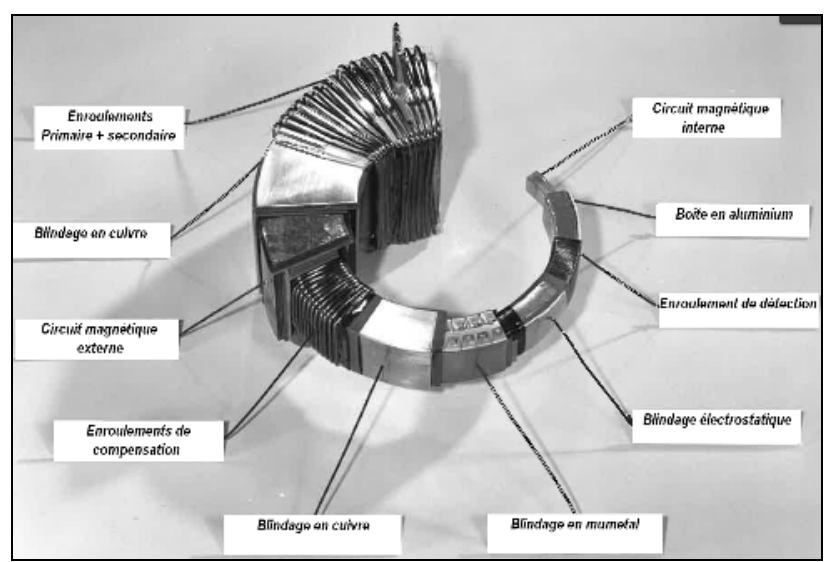

Figure1. Constitution d'un comparateur de courant

elle comporte essentiellement :

- un circuit magnétique intérieur en perminphyl.

-un enroulement de détection entourant le circuit magnétique intérieur, son rôle est de détecter la condition d'annulation de la tension induite apparaissant entre ces bornes.

-un écran magnétique en mumétal entourant l'enroulement de détection et le circuit magnétique intérieur.

-un enroulement de compensations secondaire entourant tous les éléments précédents. Il est de même nombre de spires et de même couplage que l'enroulement secondaire.

-un enroulement de compensation primaire entourant tous les éléments précédents. Il permet de compenser les effets des capacités parasites de la source de courant primaire. -un enroulement de compensation complémentaire. Il permet de compenser les influences perturbatrices des capacités parasites de l'enroulement secondaire.

-un circuit magnétique extérieur entourant tous les éléments précédents.

- un enroulement secondaire entourant tous les éléments précédents.
- un enroulement primaire entourant tous les éléments précédents

En outre des écrans électrostatiques et des blindages permettent d'éliminer les couplages capacitifs directs entre les enroulements.

\subsection{Les comparateurs au LNE}

Deux comparateurs de courant à rapports multiples sont conçus au Laboratoire Centrale des Industries Electriques dans les années 1970 (figure.2) pour servir de référence métrologique notamment pour l'étalonnage des transformateurs de courant et des shunts aux fréquences industrielles $50 \mathrm{~Hz}, 60 \mathrm{~Hz}$ et $400 \mathrm{~Hz}$. Le premier dénommé "petit comparateur » pour les rapports de 1 à 200 et le deuxième dénommé « grand comparateur » pour les rapports de 5 à 5000 . Pour un courant secondaire de 5 A les courants nominaux s'étendent à $1 \mathrm{kA}$ pour le petit comparateur et à $25 \mathrm{kA}$ pour le grand comparateur. L'association en cascade des deux comparateurs peut permettre d'étendre la gamme des rapports à 10000 en vue de traiter des courants en régime sinusoïdal jusqu'à 50 kA, voire au delà.

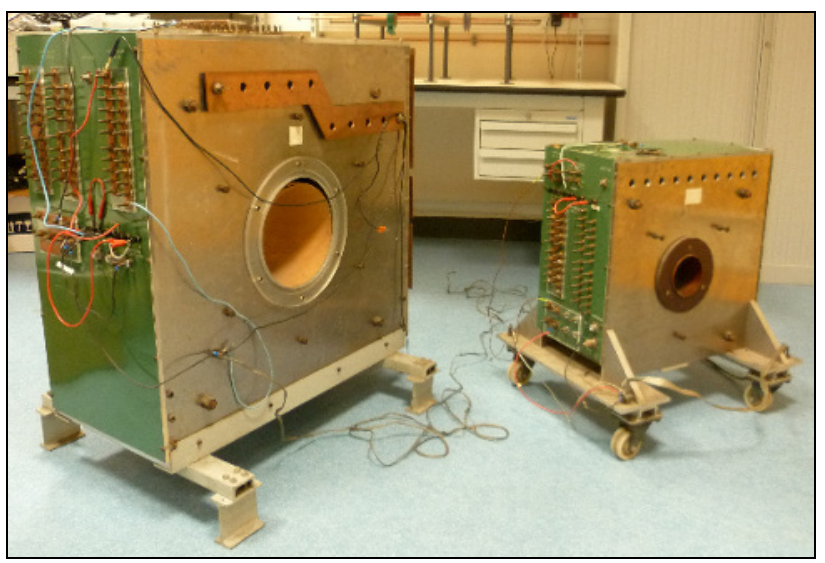

Figure2. Les comparateurs de courant au LNE

\subsection{Principe de fonctionnement}

Le principe de fonctionnement du comparateur est présenté dans la figure 3 . Les courants primaire et secondaire du transformateur à étalonner traversent des enroulements du comparateur de même rapport $\mathrm{N}_{2} / \mathrm{N}_{1}$. L'enroulement de détection permet de détecter la nullité de la somme des flux magnétiques provoqués par les courants traversant les enroulements supérieurs.

Les erreurs magnétiques et capacitives du comparateur sont inférieures à $1.10^{-6}$ en valeur relative, la tension prélevée aux bornes de l'enroulement de détection doit être nulle en présence d'un transformateur en étalonnage parfait (sans erreur). Un transformateur imparfait apporte une erreur affectant la tension aux bornes de l'enroulement de détection, l'annulation de cette tension est obtenue par l'injection en phase et en quadrature du courant $\mathrm{I}_{\mathrm{e}}$. Ce dernier traduit directement le courant d'erreur du transformateur en étalonnage. 


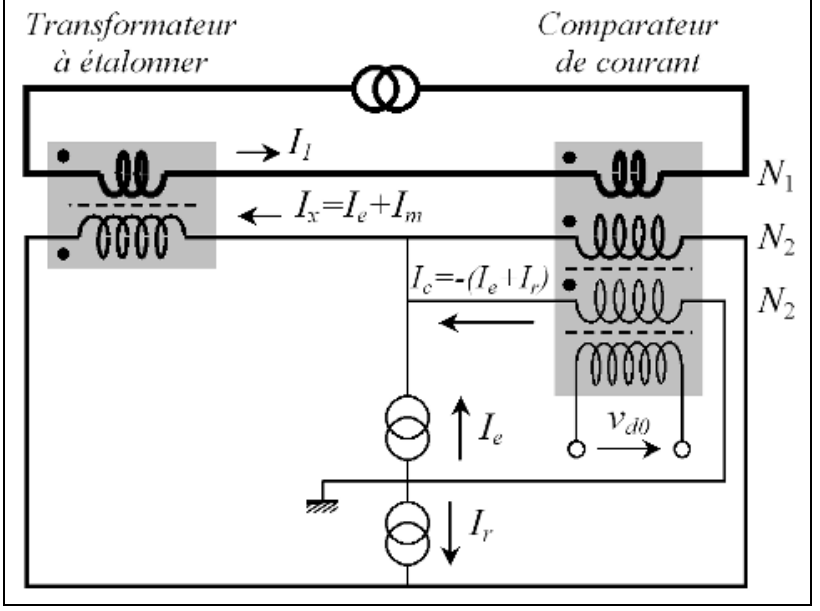

Figure 3. Principe de fonctionnement du comparateur

le courant magnétisant le comparateur $I_{r}$ est fourni par une autre source d'appoint, il traverse à flux soustractif l'enroulement secondaire et l'enroulement de compensation secondaire et il n'a aucun effet métrologique sur le flux aux bornes de l'enroulement de détection. Il est réglé de façon à donner la valeur désirée à la charge du transformateur à étalonner, définie par le quotient de la tension à ses bornes secondaires $\mathrm{V}_{\mathrm{x}}$ par l'intensité de son courant secondaire $\mathrm{I}_{\mathrm{x}}$ :

$$
Z=\frac{V_{x}}{I_{x}}
$$

Pour assurer commodément le rôle de ces deux générateurs, deux dispositifs auxiliaires ont été mis au point, il s'agit de dispositif de charge fictive et de dispositif d'injection de courant d'erreur.

\subsection{Principe du dispositif de charge fictive}

Le dispositif de charge fictive fonctionne comme un système d'asservissement fournissant automatiquement à sa sortie le courant $I_{r}$ nécessaire pour asservir la tension à la valeur Z.I $_{\mathrm{x}}$. A cet effet il comprend trois parties fonctionnelles auxquelles correspondent respectivement trois portes :

- une porte d'entrée qui sert à introduire le signal de référence proportionnel à $I_{x}$. Elle comporte tous les organes de réglages permettant d'ajuster et d'afficher avec exactitude et avec une grande résolution les deux composantes complexes $\mathrm{Z}$ qui caractérise la charge à obtenir.

- une porte de mesure servant à l'introduction la grandeur $\mathrm{V}_{\mathrm{x}}$ à asservir, suivi d'un circuit d'adaptation de cette grandeur élaborant le signal de mesure.

- une porte de sortie fournissant le courant $I_{r}$, raccordée aux enroulements du comparateur par lesquels s'exerce l'action sur la grandeur $\mathrm{V}_{\mathrm{x}}$ et qui assurent, conjointement avec la partie d'adaptation du signal $\mathrm{V}_{\mathrm{x}}$, la contre réaction du système.

\subsection{Principe du dispositif d'injection}

Le dispositif d'injection fonctionne comme un amplificateur opérationnel à gain en boucle ouverte, la contre réaction est assurée par le couplage inductif entre l'enroulement de compensation secondaire du comparateur et l'enroulement de détection de façon à déterminer automatiquement le courant d'injection nécessaire pour asservir à une très faible valeur la tension aux bornes de l'enroulement de détection. A cet effet, il comporte trois parties fonctionnelles auxquelles correspondent trois portes :

- une porte comprenant tous les organes d'adaptation et de réglages élaborant avec exactitude et une grande résolution, à partir du signal référence proportionnel à $\mathrm{I}_{\mathrm{x}}$, les deux composantes du facteur complexe $A$ qui caractérise la proportionnalité du courant $I_{x}$ au courant $I_{e}$ et qui, lorsque le signal de détection est nul, représente directement l'erreur du transformateur à étalonner.

- une porte d'entrée raccordée à l'enroulement de détection du comparateur permettant de prélever et de contrôler la tension à ses bornes.

- une porte de sortie raccordée à l'enroulement de compensation secondaire du comparateur et fournissant le courant $\mathrm{I}_{\mathrm{e}}$ après transformation et amplification de sortie.

\section{Les nouveaux dispositifs}

Un nouvel équipement électronique a été conçu en remplacement des anciens, dans lequel un asservissement logiciel remplace le système d'asservissement à lecture directe, voir paragraphe 2.3 et 2.4. Ce nouvel équipement est entièrement contrôlable à distance par un ordinateur via une connexion USB, ce qui permet une automatisation complète : il n'y a plus aucune commande manuelle (commutateurs ou potentiomètres) car elles ont toutes été remplacées par des circuits électroniques.

\subsection{Le dispositif de charge fictive à asservissement logiciel.}

La tension $\mathrm{V}_{\mathrm{x}}$ apparaissant aux bornes du secondaire du transformateur à étalonner (Fig. 4) est contrôlée en appliquant une tension $\mathrm{E}_{\mathrm{r}}$ entre les enroulements secondaire et de compensation du comparateur connectés en série. Le courant $I_{r}$ qu'elle fait circuler est sans effet sur la tension détectée $\mathrm{V}_{\mathrm{d}}$.

La tension $E_{r}$ est liée au courant secondaire $I_{x}$ en utilisant un opérateur vectoriel dit d'action dont la transmittance est réglée pour obtenir la tension $\mathrm{V}_{\mathrm{x}}$ désirée. Pour cela, la tension $\mathrm{R}_{\mathrm{s}} \cdot \mathrm{I}_{\mathrm{x}}(\mathrm{c}+\mathrm{jd})$ sortant de l'opérateur vectoriel est comparée, à l'aide d'un détecteur synchrone, à celle issue de l'enroulement secondaire du transformateur en étalonnage.

L'opérateur d'action est suivi d'un amplificateur $\mathrm{A}_{2}$ qui dans certains cas doit pouvoir fournir ou absorber une puissance importante.

\subsection{Le dispositif d'injection à asservissement logiciel}

Le principe de dispositif d'injection à asservissement logiciel est présenté dans la figure 5. Le principe consiste 


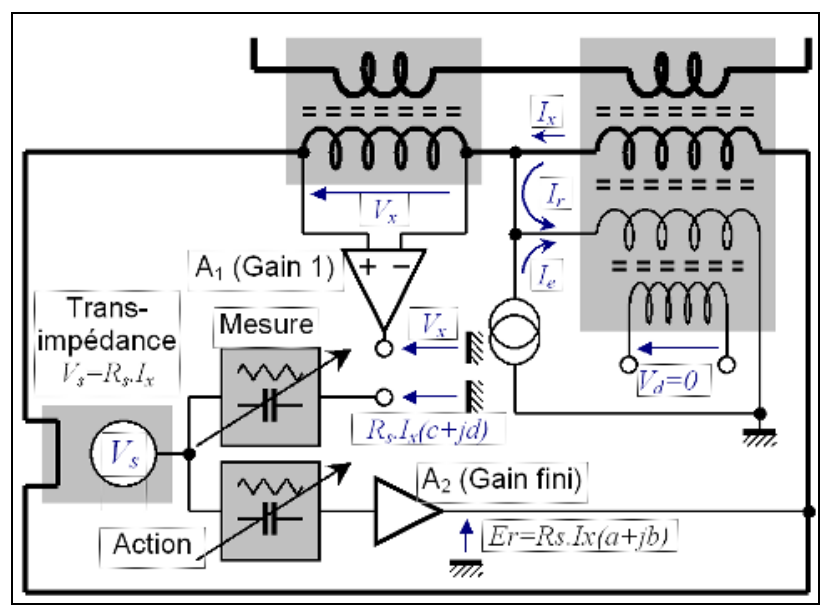

Figure 4. Principe de dispositif de charge fictive à asservissement logiciel

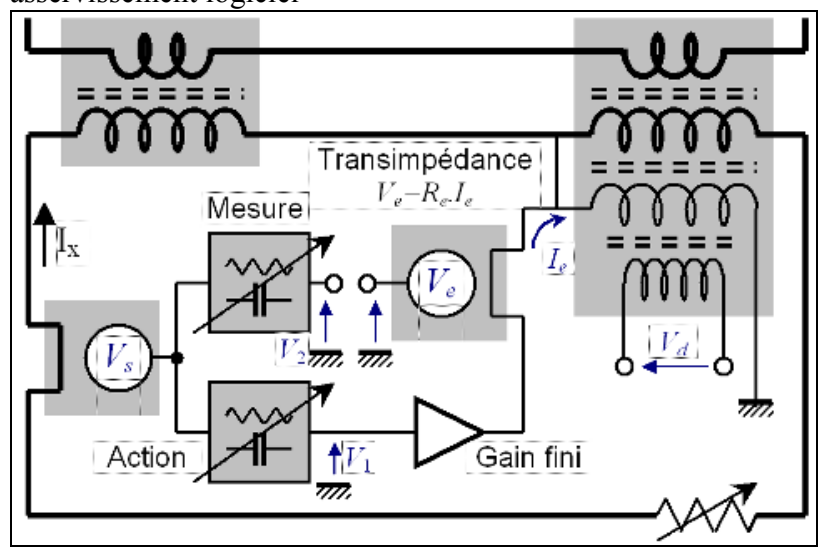

Figure 5. Principe de dispositif d'injection à asservissement logiciel

à utiliser des opérateurs vectoriels constitués au moyen d'un signal de référence proportionnel à $I_{x}$.

Un premier opérateur vectoriel d'action est réglé pour annuler la tension $\mathrm{V}_{\mathrm{d}}$ contrôlée au moyen d'un détecteur synchrone. Le courant injecté $\mathrm{I}_{\mathrm{e}}$ est mesuré en faisant l'opposition de son image $\mathrm{V}_{\mathrm{e}}$ au travers d'une transimpédance connue avec la tension de sortie d'un deuxième opérateur vectoriel de mesure.

\subsection{Interconnexion de l'ensemble}

Les comparateurs de courant sont des dispositifs très lourds et volumineux, les câbles ou barres portant le courant primaire sont susceptibles de rayonner des champs importants. Les éléments d'électronique sont donc nécessairement dans un coffret situé à quelque distance, et il faut minimiser le nombre de connexions entre celui-ci et les éléments extérieurs. La figure 6 fait l'inventaire des interconnexions, en montrant par où est connectée la terre. Le comparateur de courant, le transformateur à étalonner ont leurs bâtis et leurs écrans connectés à la terre, mais aucun enroulement ne l'est directement. C'est par le coffret de l'équipement électronique qu'ils peuvent l'être. Le boîtier du transformateur de mesure du courant secondaire est à la

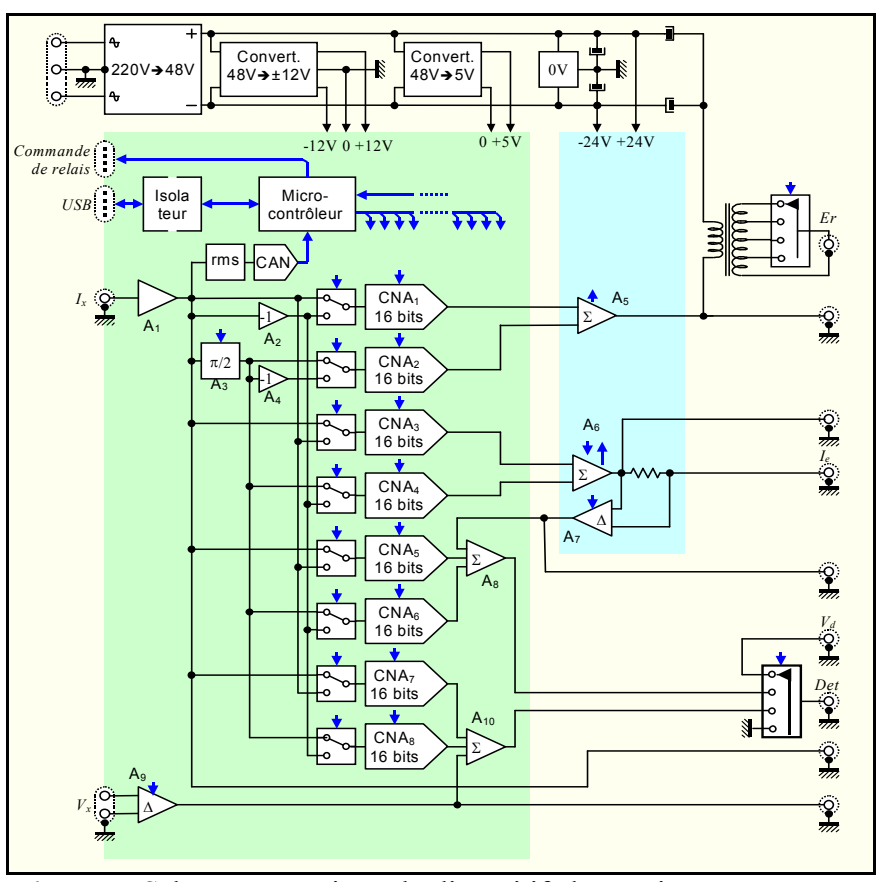

Figure 7. Schéma synoptique du dispositif électronique

terre par sa liaison de télécommande. Le schéma synoptique est présenté dans la figure 7.

Chacun des éléments constitutifs du dispositif ont été évalués. Les convertisseurs numériques analogiques sont au cœur du système et les paramètres essentiels sont leur résolution, leur non-linéarité et leur comportement en fréquence. La dissipation de l'amplificateur sur une charge a été considérée suivant que la charge absorbe ou dissipe de l'énergie, le courant d'erreur injecté a été mesurés sur 2 valeurs de résistance de mesure.

La modernisation radicale a également pour conséquence une réduction importante du volume (le tout tient dans un coffret standard 19 pouces $\mathrm{x} 3 \mathrm{U}$ ).

Le logiciel associé apporte une aide importante dans le choix d'une configuration parmi les nombreuses qu'offrent les deux comparateurs, et montre clairement les connexions à établir (Figures 8 et 9). Il a été développé avec LabVIEWTM.

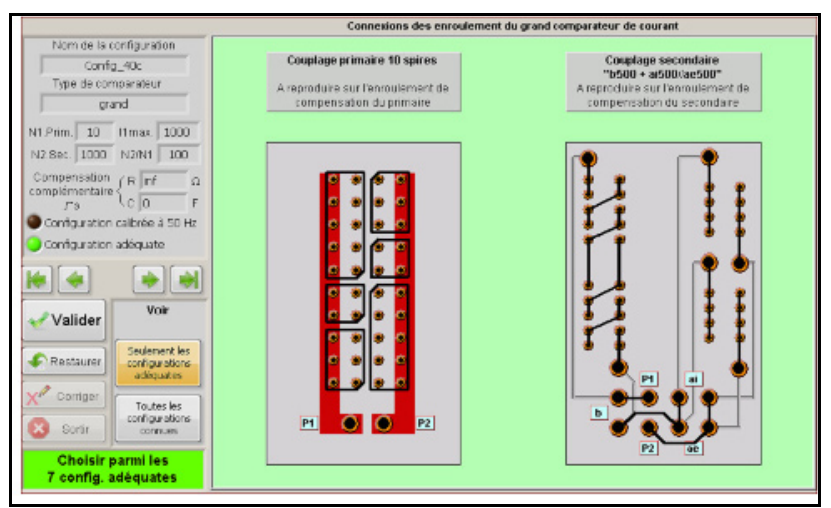

Figure 8. Choix des configurations apporté par le logiciel. 


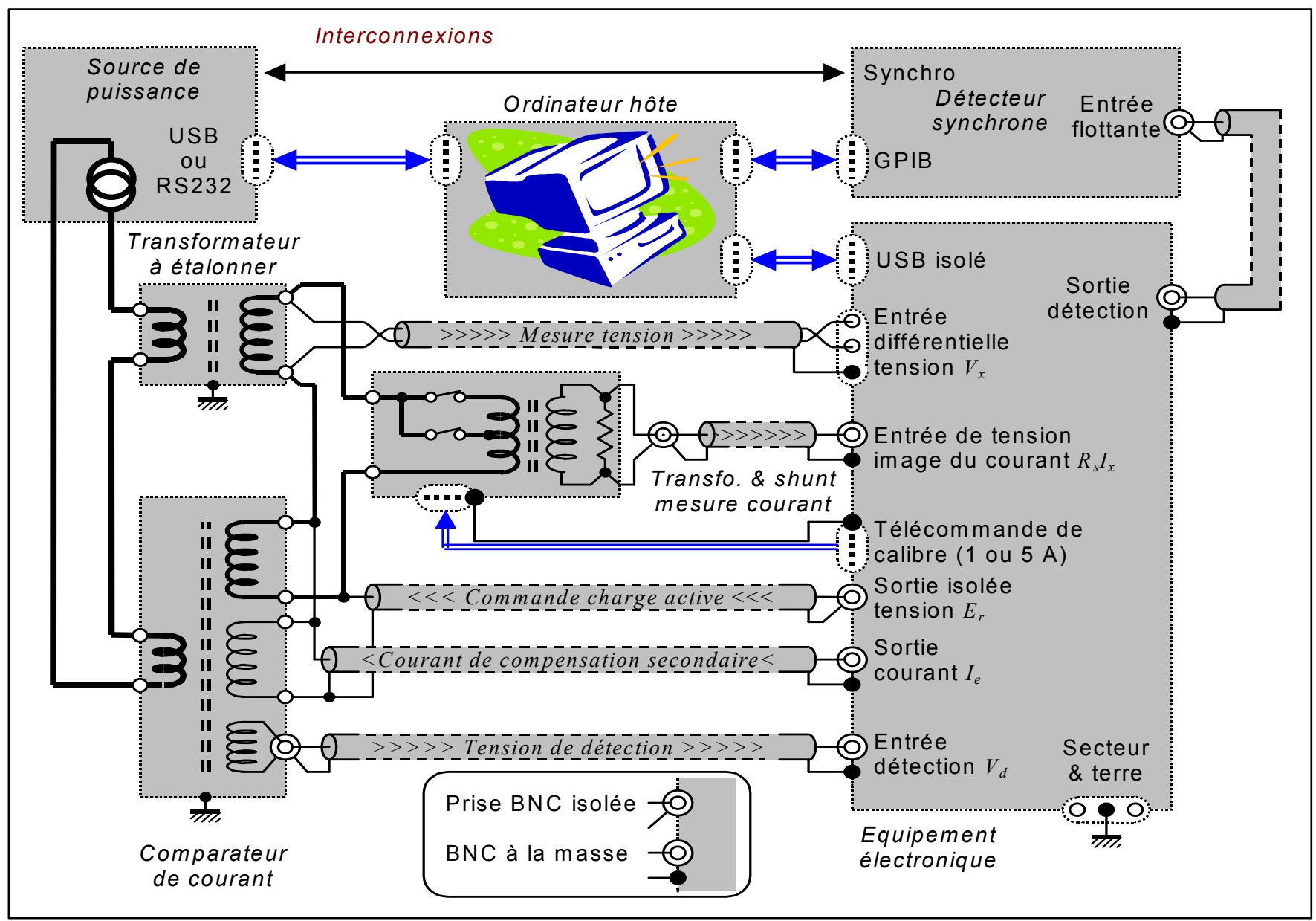

Figure 6. Interconnexion de l'ensemble(comparateur, dispositif de charge fictive, dispositif d'injection de courant d'erreur et logiciel)

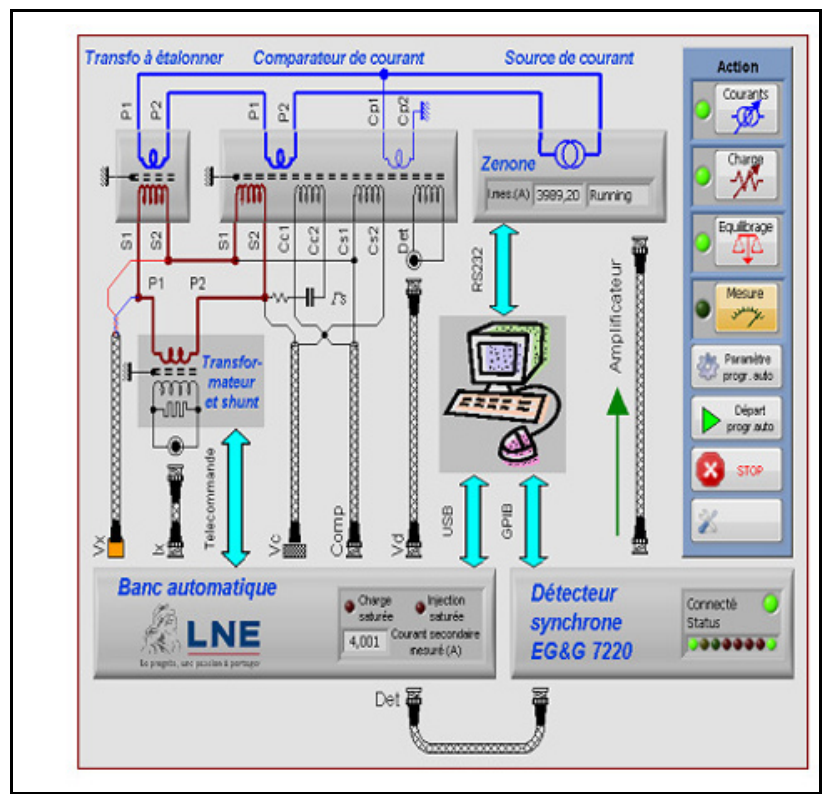

Figure 9. Fenêtre de démarrage.

\section{Conclusions et perspectives}

Les expérimentations du nouveau dispositif électronique dans un montage d'étalonnage d'un transformateur de courant de forte intensité sont en cours de réalisation. Ce nouveau dispositif permettra une automatisation complète des mesures et une amélioration de la stabilité des étalonnages grâce à la puissance de l'asservissement logiciel.

\section{Références}

1. R Knosp, «L'emploi de comparateurs de courants alternatifs dans la métrologie des courants de forte intensité »- RGE 5/81 pp. 350-357.

2. N.L. Kusters and W.M.Moore «The Compensated Current Comparator. A New Reference Standard for Current Transformer Calibration in Industry » - 1964 3. N.L. Kusters and W.J.M. Moore «A phantom burden for current transformer calibration », National research council of Canada Ottawa, Ontarion, Canada..1973 